\title{
Development and characterization of human-induced pluripotent stem cell-derived cholangiocytes
}

\author{
Thiago M De Assuncao ${ }^{1,2,7}$, Yan Sun 1,2,7, Nidhi Jalan-Sakrikar ${ }^{1,2}$, Mary C Drinane ${ }^{1,2}$, Bing Q Huang ${ }^{3}$, Ying Li $i^{4}$, \\ Jaime I Davila ${ }^{4}$, Ruisi Wang ${ }^{1,2}$, Steven P O'Hara ${ }^{3}$, Gwen A Lomberk ${ }^{1,2,5}$, Raul A Urrutia ${ }^{1,2,5}$, Yasuhiro Ikeda ${ }^{6}$ and \\ Robert C Huebert ${ }^{1,2,5}$
}

Cholangiocytes are the target of a heterogeneous group of liver diseases known as the cholangiopathies. An evolving understanding of the mechanisms driving biliary development provides the theoretical underpinnings for rational development of induced pluripotent stem cell (iPSC)-derived cholangiocytes (iDCs). Therefore, the aims of this study were to develop an approach to generate iDCs and to fully characterize the cells in vitro and in vivo. Human iPSC lines were generated by forced expression of the Yamanaka pluripotency factors. We then pursued a stepwise differentiation strategy toward iDCs, using precise temporal exposure to key biliary morphogens, and we characterized the cells, using a variety of morphologic, molecular, cell biologic, functional, and in vivo approaches. Morphology shows a stepwise phenotypic change toward an epithelial monolayer. Molecular analysis during differentiation shows appropriate enrichment in markers of iPSC, definitive endoderm, hepatic specification, hepatic progenitors, and ultimately cholangiocytes. Immunostaining, western blotting, and flow cytometry demonstrate enrichment of multiple functionally relevant biliary proteins. RNA sequencing reveals that the transcriptome moves progressively toward that of human cholangiocytes. iDCs generate intracellular calcium signaling in response to ATP, form intact primary cilia, and self-assemble into duct-like structures in three-dimensional culture. In vivo, the cells engraft within mouse liver, following retrograde intrabiliary infusion. In summary, we have developed a novel approach to generate mature cholangiocytes from iPSCs. In addition to providing a model of biliary differentiation, iDCs represent a platform for in vitro disease modeling, pharmacologic testing, and individualized, cell-based, regenerative therapies for the cholangiopathies. Laboratory Investigation (2015) 95, 684-696; doi:10.1038/labinvest.2015.51; published online 13 April 2015

Biliary diseases continue to be the cause of significant morbidity and mortality, in both children and adults. ${ }^{1}$ Cholangiocytes, the specialized epithelial cells lining the intra- and extrahepatic bile ducts, are the target of a heterogeneous group of disorders known as the cholangiopathies. $^{2}$ The obliterative cholangiopathies are a subset of these disorders that have, as a hallmark, progressive cholangiocyte destruction, culminating in ductopenia and cholestasis. Cholangiocytes also suffer damage during and after liver transplantation in the form of preservation injury, cellular rejection, disease recurrence, and ischemic cholangiopathy. ${ }^{3,4}$ Most etiologies of the obliterative cholangiopathies result in progressive biliary fibrosis culminating in end-stage liver disease that is essentially untreatable without liver transplantation. However, an inadequate supply of donor organs limits the effectiveness of this surgical approach. Given the targeted cellular destruction typical of the cholangiopathies, these diverse disorders may be amenable to cell replacement strategies in these varying circumstances. Therefore, the biliary system is an attractive target for cellbased regenerative medicine approaches to study and potentially treat the disorders.

Although the liver has remarkable intrinsic regenerative properties, this mechanism is impaired in the setting of chronic liver disease. ${ }^{5}$ Explosive growth in the field of liver regenerative medicine, including hepatic differentiation of induced pluripotent stem cells (iPSC), has the potential to provide a new platform for the study and treatment of liver

\footnotetext{
${ }^{1}$ Division of Gastroenterology and Hepatology, Mayo Clinic and Foundation, Rochester, MN, USA; ${ }^{2}$ Gastroenterology Research Unit, Mayo Clinic and Foundation, Rochester, MN, USA; ${ }^{3}$ Center for Basic Research in Digestive Diseases, Mayo Clinic and Foundation, Rochester, MN, USA; ${ }^{4}$ Division of Biomedical Statistics and Informatics, Mayo Clinic and Foundation, Rochester, MN, USA; ${ }^{5}$ Center for Cell Signaling in Gastroenterology, Mayo Clinic and Foundation, Rochester, MN, USA and ${ }^{6}$ Department of Molecular Medicine, Mayo Clinic and Foundation, Rochester, MN, USA

Correspondence: Dr RC Huebert, MD, Gastroenterology Research Unit, Mayo Clinic and Foundation, 200 First Street SW, Rochester, MN 55905, USA.

E-mail: huebert.robert@mayo.edu

${ }^{7}$ These authors contributed equally to this work.
}

Received 14 November 2014; revised 26 January 2015; accepted 4 February 2015 
disorders that could ultimately transform the care of patients with end-stage liver disease. ${ }^{6}$ The newly discovered ability of the Yamanaka factors to reprogram somatic cells to pluripotency has revealed remarkable cellular plasticity and, indeed, it is now possible to generate iPSCs from virtually any tissue in the human body and to recapitulate developmental biology in vitro, to generate diverse cellular phenotypes. ${ }^{7}$ Based on emerging details governing developmental biology of the liver, ${ }^{8}$ a number of groups have developed various methods for generating hepatocyte-like cells from iPSCs via stepwise differentiation strategies ${ }^{9-19}$ or by direct differentiation from fibroblasts. ${ }^{20,21}$ Although some of these protocols described biliary elements, pluripotent stem cell-derived cholangiocytes had not been directly nor extensively studied until very recently when cholangiocytes were developed from embryonic stem cells and bipotent HepaRG cells, ${ }^{22}$ an approach that was also effective in iPSCs. Simultaneously, our group and others have begun to develop additional targeted approaches to create iPSC-derived cholangiocytes (iDCs). New understanding of the mechanisms driving biliary development ${ }^{23-26}$ and cellular plasticity during liver regeneration/repair ${ }^{27,28}$ have provided the theoretical underpinnings for the rational development and use of iDCs as individualized disease models and potentially as regenerative therapeutics for biliary disease. ${ }^{29}$ Furthermore, this direction is conceptually appealing given the clinical access to the biliary tree in humans afforded by endoscopic retrograde cholangiopancreatography, a technique readily available at every major academic medical center in the world.

As hepatocytes and cholangiocytes share common precursors and as biliary differentiation pathways are now being more fully elucidated, we reasoned that targeted modifications to existing differentiation strategies should allow for generation of iDCs. This study provides technical and conceptual innovations by demonstrating that human myofibroblast-derived iPSCs can be reproducibly differentiated toward an adult bile duct epithelial fate, expressing numerous markers of functionally mature cholangiocytes. RNA sequencing at each phase of differentiation followed by principal component analysis and differential expression analysis confirm that the transcriptome is gradually altered from iPSC toward that of human cholangiocytes. In addition, the transcriptional profiles during the iPSC to iDC transition appear to recapitulate several aspects of biliary development. We go on to demonstrate that iDCs form primary cilia on their apical surface, have intact calcium signaling, and form duct-like structures in three-dimensional (3D) culture. Furthermore, we show for the first time that stem cellderived cholangiocytes can engraft within the liver in vivo after retrograde intrabiliary infusion in a mouse model. The experimental results from this study provide new information regarding the feasibility of iPSC-based differentiation strategies targeting cholangiocytes and produce the foundation for a detailed dissection of the mechanisms governing biliary differentiation. Furthermore, mature iDCs derived from patient-specific iPSCs could potentially be a limitless source of high-quality, individualized cells that could be: (1) studied in vitro as patient-specific models of biliary disease; (2) treated in vitro to test putative therapeutic compounds; (3) genetically modified to correct underlying disease-causing defects; and (4) transplanted (without the need for immunosuppression) as individualized, cell-based, regenerative therapeutics for the cholangiopathies.

\section{MATERIALS AND METHODS Cholangiocyte Culture}

iDC, H69, and NHC were cultured in H69 media (DMEM/ F12 supplemented with $10 \%$ fetal bovine serum (FBS), 1\% penicillin/streptomycin, adenine, insulin, epinephrine, T3-T, hydrocortisone, and epidermal growth factor).

\section{Reprogramming/iPSC Culture}

Human myofibroblasts were cultured from biopsy specimens with appropriate IRB oversight and informed consent. These cells were induced to pluripotency by transient forced expression of OCT4, SOX2, KLF4, and c-MYC, using the Sendai system, as previously described. ${ }^{30}$ iPSC were seeded in culture plates pre-coated with $0.1 \%$ Matrigel (R\&D Systems) and cultured in nutristem XF/FF culture medium (Stemgent) supplemented with $16 \%$ mTeSR1 basal medium (Stemcell Technologies), mTeSR $15 \times$ Supplement, $50 \times$ StemGS (ScienCell Research Laboratories) and 1\% penicillin/ streptomycin.

\section{Generation of iDC}

The stepwise differentiation toward iDCs was accomplished by a process of temporal exposure to biliary morphogens (Figure 1a). iPSC were induced to definitive endoderm (DE) for 4 days using Advanced RPMI medium changed and supplemented daily with addition of $50 \mathrm{ng} / \mathrm{ml}$ Activin A and $50 \mathrm{ng} / \mathrm{ml}$ Wnt3A in the presence of Matrigel. DE cells were treated daily with $10 \mathrm{ng} / \mathrm{ml}$ fibroblast growth factor 2 (FGF2), $20 \mathrm{ng} / \mathrm{ml}$ bone morphogenic protein 4 (BMP4) and $50 \mu \mathrm{g} / \mathrm{ml}$ Sonic hedgehog (SHH) for 4 days, for hepatic specification (HS). HS cells were induced to hepatic progenitor (HP) cells for 4 days with daily exposure to $50 \mu \mathrm{g} / \mathrm{ml} \mathrm{SHH}$ and $100 \mu \mathrm{g} /$ $\mathrm{ml}$ recombinant Jagged-1. To generate iDC, HP cells were treated for 4 days with $\mathrm{H} 69$ media changed and supplemented daily with $100 \mu \mathrm{g} / \mathrm{ml}$ transforming growth factor- $\beta$ (TGF- $\beta$ ) and replacement of Matrigel with collagen. All growth factors were purchased from R\&D Systems (Supplementary Table 1).

\section{Quantitative RT-PCR}

Total RNA was extracted from human iPSC, DE, HS, HP, and iDC cells using the RNeasy Plus Mini Kit (Qiagen). Reverse transcription was performed with $5 \mu \mathrm{g}$ RNA, using oligo $(\mathrm{dT})$ primer and SuperScript III. Real-time PCR was performed in a volume of $25 \mu \mathrm{l}$ using Sybr Green Master Mix and the 7500 Real-Time PCR System (Applied Biosystems). Primer sequences are shown in Supplementary Table 2. 

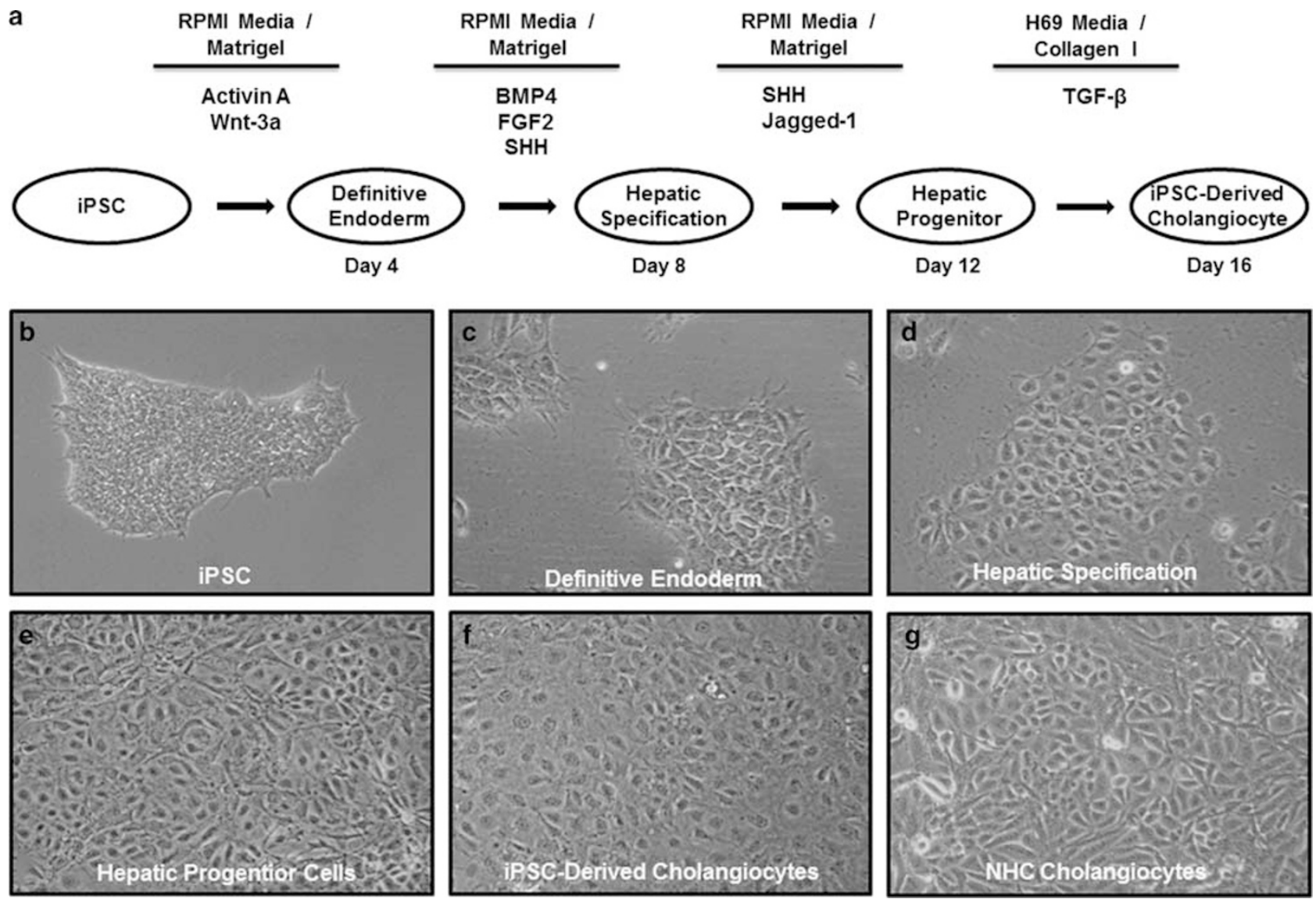

Figure 1 Protocol for generation of induced pluripotent stem cell (iPSC)-derived cholangiocyte (iDC) from iPSC and morphologic analysis. (a) The protocol proceeds through phases of definitive endoderm (DE), hepatic specification (HS), and hepatic progenitor (HP), and incorporates defined culture media, precise extracellular components, and temporal exposure to key biliary morphogens. Light microscopy $(\times 10)$ shows phenotypic changes at each stage of differentiation with the transformation of $(\mathbf{b})$ the stem cell phenotype towards (c- $\mathbf{f})$ an epithelial monolayer, similar to the $(\mathbf{g}) \mathrm{NHC}$ cholangiocyte cell line.

\section{Western Blotting}

Protein isolation was done with lysis buffer. Concentration was determined using a spectrophotometer. Twenty-five micrograms of protein was loaded onto $4-12 \%$ Tris-Glycine gels, electrophoresed, and transferred onto nitrocellulose membranes (Scientific Laboratory Supplies) for blotting. The membrane was blocked, incubated with primary antibodies, rinsed with TBST, and incubated with secondary antibodies (Supplementary Table 3). After washing, the membrane was exposed to luminol (Santa Cruz Biotechnology) and developed.

\section{Immunofluorescence Microscopy}

Cells were seeded on glass coverslips coated with Matrigel. For observation of primary cilia, some cells were grown in monolayer for $\sim 7$ days followed by serum starvation to induce cilia. After fixation with paraformaldehyde and permeabilization with Triton X-100, cells were blocked with FBS, incubated with primary antibodies and then with fluorescence-conjugated secondary antibodies. Cells were washed and nuclei were stained with 4'6-diamidino-2phenylindole dihydrochloride (Sigma). Coverslips were mounted onto glass slides and observed under confocal microscopy (Carl Zeiss, Jena, Germany). Images were acquired using Zeiss LSM software.

\section{Flow Cytometry}

Fluorescence-activated cell sorting (FACS) was performed in the Mayo Flow Cytometry Laboratory, as previously described. ${ }^{31}$ Briefly, one million cells were treated with a Trypsin digestion solution and fixed in $4 \%$ paraformaldehyde for 20 min followed by three washes in FACS buffer (2\% FBS + DPBS). Blocking was performed in $0.5 \%$ BSA in FACS buffer for $30 \mathrm{~min}$ at room temperature. The cell suspension was centrifuged at $300 \mathrm{~g}$ for $5 \mathrm{~min}$ and the supernatant was removed by aspiration. Cells were incubated with primary antibody on ice for $30 \mathrm{~min}$, washed three times, stained with fluorochrome-conjugated secondary antibodies, in the dark, on ice, for $60 \mathrm{~min}$, and washed another three times. Cells were analyzed on a FACSCalibur analytical flow cytometer in three 
independent sessions. Data files were analyzed using Cellquest software.

\section{RNA Sequencing}

RNA sequencing was conducted in collaboration with the Mayo Medical Genomics Facility. From each phase of differentiation (iPSC, DE, HS, HP, and iDC), from cholangiocyte cell lines (H69 and NHC), and from isolated human cholangiocytes (iHCs), total RNA was isolated. Quality of the RNA was assessed by the Mayo Gene Expression Core using Agilent Bioanalyzers. All samples had RNA Integrity Numbers $>$ 7.0. RNA sequencing was performed as paired-end base reads on an Illumina HiSeq 2000, with three samples per lane, using the TruSeq SBS Sequencing Kit, Version 3. Base calling was performed using Illumina's RTA version 1.12.4.2. Bioinformatics were performed with the assistance of the Mayo Division of Biostatistics and Informatics. Analysis of each sample (alignment statistics, in-depth quality control metrics, and gene and exon expression levels) was done using Mayo Clinic's MAPRSeq v1.2 (ref. 32). Reads were mapped using Tophat version 2.0.6 (ref. 33) against the hg19 reference genome and gene counts were produced using htseq. Subsequent principal component analysis was performed using Partek Genomics Suite version 6.6 (Partek, St. Louis, $\mathrm{MO}$ ). Differential expression analyses between samples were computed using an edgeR version 3.3.8 algorithm. ${ }^{34}$

\section{D Culture}

Cells were suspended in a mixture of $1 \times$ DMEM, $\mathrm{NaOH}$ $(10 \mathrm{nM})$, type I collagen $(2.0 \mathrm{mg} / \mathrm{ml})$ and $33 \%$ Matrigel. Cells $\left(1 \times 10^{4}\right)$ were added to glass-bottom culture dishes. The mixture solidified and was overlaid with media. Plates were cultured for 7 days and assessed by light and immunofluorescence microscopy.

\section{Intracellular $\mathrm{Ca}^{2+}$ Signaling}

iDC and NHC cells were plated in H69 media. After $24 \mathrm{~h}$, cells were serum starved for $2 \mathrm{~h}$ and incubated with Fluo-4AM (Invitrogen) in Hank's balanced salt solution supplemented with $20 \mathrm{mM}$ HEPES and $2.5 \mathrm{mM}$ Probenecid for $60 \mathrm{~min}$. A FlexStation III (Molecular Devices) automated plate reader was used to measure calcium flux. After establishing baseline fluorescence for $30 \mathrm{~s}$, ATP (ACROS Organics) or vehicle $(10 \mu \mathrm{l})$ were added and fluorescence was further read for 2 min.

\section{Electron Microscopy}

Scanning electron microscopy was performed as previously described. ${ }^{35}$ iDC were grown in a monolayer for $\sim 7$ days followed by $24 \mathrm{~h}$ of serum starvation, fixed in glutaraldehyde, post fixed in osmium tetroxide, dehydrated, critical-point dried, sputter coated, and imaged with a scanning electron microscope (Hitachi) at $40000 \times$.

\section{Retrograde Intrabiliary Infusion}

Animals were anesthetized using a combination of ketamine and xylazine. Using a dissecting microscope, the common bile duct was temporarily occluded (for a total of $2 \mathrm{~h}$ ) with a vascular clamp. The gallbladder was dissected from the liver (Supplementary Figure 1A) and two silk sutures were placed around the cystic duct (Supplementary Figure 1B). The gallbladder and cystic duct were cannulated with a 24-guage, $3 / 4$ " angiocatheter (Supplementary Figure 1C) and sutured in place (Supplementary Figure 1D). Cells $\left(10^{6}\right)$ were injected at a flow rate of $0.2 \mathrm{ml} / \mathrm{min}$ (Supplementary Figure 1E). Some cells were labeled with green fluorescent protein (GFP), using lentiviral transduction, and selected with 2 weeks of exposure to puromycin. After infusion, a silk tie was placed between the gallbladder and the cystic duct, and a cholecystectomy was completed. At $2 \mathrm{~h}$ after clamping, the common bile duct was unclamped and the animals were closed using interrupted chromic gut sutures. At day 3 after cell transplantation, liver was collected, processed with paraffin embedding and sectioning, and assessed for cell engraftment by immunostaining for the human-specific class 1 major histocompatibility complex (MHC1) and cytokeratin 7 (CK7), by confocal microscopy for GFP and by immunofluorescence for CK7 and MHC1.

\section{Statistical Analysis}

Data are presented as the mean \pm s.e. Data represent typical experiments reproduced at least three times. Analysis was performed using Graph Stat Prism software (GraphPad Software, La Jolla, CA). Statistical analyses used one-way analysis of variance with a Tukey's post test.

\section{RESULTS}

\section{Generation of iDC}

Human myofibroblasts were induced to pluripotency by the transient expression of the transcription factors (OCT4, SOX2, KLF4, and c-MYC) using the Sendai system. The iPSC had typical morphologic features of iPSC including discrete smooth-edged colonies and high nuclear-to-cytoplasmic ratio (Figure 1b). They also stained positive for numerous markers of pluripotency including TRA-1-60, OCT4, SOX2, NANOG, and alkaline phosphatase (Supplementary Figure 2A), and have gene expression profiles distinct from the parental fibroblasts but highly similar to a lentiviral-reprogrammed iPSC clone from keratinocytes and to a human embryonic stem cell line (Supplementary Figure 2B). As hepatocytes and cholangiocytes share a common precursor, we postulated that subtle, but targeted, alterations to existing hepatocyte differentiation protocols should allow us to develop iDCs. We noticed that prior reports that had included $\mathrm{SHH},{ }^{13}$ Notch signaling, ${ }^{36}$ or TGF- $\beta^{37}$ had also reported some resulting biliary elements and thus these morphogens were included in our protocol. In addition, based on increasing fundamental knowledge of developmental biology of the biliary system ${ }^{25}$ as well as liver regeneration, ${ }^{26}$ we further 
refined the novel stepwise protocol for the in vitro differentiation of iPSCs to iDCs (Figure 1a). We also avoided the hypoxia present in many hepatocyte differentiation protocols, as cholangiocytes are sensitive to hypoxia. ${ }^{38}$ The differentiation protocol was performed in defined media (Advanced RPMI) with feeder-free culture conditions. Initially, iPSC on Matrigel were treated daily with Activin A and Wnt3a in RPMI for 4 days, for induction of DE. DE cells were exposed daily to FGF2, BMP4, and to SHH for 4 days, for HS. HP were induced via further daily exposure to $\mathrm{SHH}$ and Jagged-1 for 4 days. For the final stage of cholangiocyte maturation, the cells were exposed daily to TGF- $\beta$, RPMI media was replaced with H69 media (a hormonally enriched media used to culture adult cholangiocytes), and Matrigel was replaced with collagen for the final 4 days.

\section{iDC Have Biliary Morphology}

Morphologic analysis of the cells showed a stepwise phenotypic change toward an epithelial monolayer, consistent with biliary epithelium (Figure 1b and f). At the DE stage, the cells began to lose the characteristic features of iPSC (discrete smooth-edged colonies with high nuclear-to-cytoplasmic ratio). In the ensuing steps, the cells lost their ability to form clusters and began to grow in an epithelioid monolayer with features reminiscent of cultured cholangiocytes and similar to existing cholangiocyte cell lines, such as NHC (Figure 1g). iDCs typically survive in culture between 3 and 10 passages, and can also be frozen in liquid nitrogen for storage, and then thawed and recovered for subsequent analyses.

\section{Molecular Analysis of the iPSC to iDC Transition}

Quantitative real-time PCR analysis at the various stages of differentiation revealed appropriate enrichment of markers at each respective stage of differentiation (Figure 2). iPSC show enrichment in the pluripotency markers SOX2 and NANOG (Figure 2a), which are then extinguished in the ensuing phases. However, iPSC lack significant expression of GATA4 and CXCR4, markers of the early endoderm lineage (Figure $2 \mathrm{~b}$ ), arguing against any spontaneous differentiation. In contrast, cells from the DE stage were highly enriched with these two markers. Markers of fetal liver and hepatic development such as HES1 and SALL4 were appropriately enriched in the HS phase (Figure 2c). Cells from the HP phase exhibited strong expression of hepatoblast markers, such as AFP and Prox-1 (Figure 2d). Notably, iDC lacked significant expression of these early hepatic markers, suggesting that these genes were downregulated during cholangiocyte maturation, something that has been challenging to achieve in hepatocyte differentiation protocols.

\section{iDCs Are Enriched in Biliary Markers}

Following the last step of differentiation, iDC showed high expression of multiple cholangiocyte markers including CK19, CK7, CFTR, PKD2, and AE2, by quantitative RTPCR (Figure 3a). As there is considerable variability within and among the existing cholangiocyte cell lines in terms of their expression of genes thought to be prototypical of cholangiocytes (Supplementary Figure 3A), there is no goldstandard control that expresses all of the cholangiocyte markers that are present in our iDCs. A significant increase in cholangiocyte markers begins to become apparent in the HS and HP phases, where SHH and Jagged-1 ligands, key biliary morphogens, were introduced to the protocol, suggesting that early priming events may already be initiating biliary programming at these phases. Importantly, iDC lack significant expression of pluripotency markers such as SOX2 and NANOG (Figure 2a), progenitor markers such as AFP, PROX-1 (Figure 2d), EpCAM and Tbx3 (Supplementary Figure $3 \mathrm{~B}$ ), as well as hepatocyte markers such as ABCB4, albumin, $\alpha$-1-antityrpsin, and tryptophan 2,3-dioxygenase (Figure $3 \mathrm{~b}$ ), indicating efficient differentiation and a relatively pure biliary fate. In fact, as long as $\mathrm{SHH}$ and Notch ligands are present in the protocol, we do not detect hepatocyte differentiation, even in the presence of hepatocyte morphogens, such as hepatocyte growth factor, dexamethasone, and oncostatin.

\section{iDCs Are Enriched in Cholangiocyte Proteins}

Western blotting demonstrates significant and progressive enrichment of multiple, functionally relevant biliary proteins in iDCs (Figure 4a) including acetylated $\alpha$-tubulin (a marker of primary cilia), AQP1 (a water channel), ASBT (a bile acid transporter), and SSTR-2 (a somatostatin receptor), along with CK7 and CK19 (the biliary cytokeratins). Notably, the iDC cells lack expression of the hepatocyte protein c-Met (the hepatocyte growth factor receptor). To demonstrate the homogeneity of cells following biliary differentiation, iDC were stained for $\mathrm{CK} 7$, which demonstrated robust and uniform staining. CK19 showed an appropriate intracellular and reticular pattern (Figure $4 \mathrm{~b}$ ), again suggesting efficient differentiation. To assess the overall enrichment of cholangiocyte proteins in iDCs, we performed flow cytometry for typical cholangiocyte markers. Flow cytometry analyses reveals that $\mathrm{iDC}$ cells were highly enriched in several cholangiocyte markers (Figure 4c) including CK7 (66.91\%, compared with $73.25 \%$ in NHC and $2.04 \%$ in HepG2), CFTR (77.34\%, compared with $22.29 \%$ in NHC and $10.47 \%$ in HepG2), and CK19 (85.3\%, compared with $46.2 \%$ in NHC and $9.22 \%$ in HepG2). iDC cells also lack expression of EpCAM $(2.03 \%$, compared with $2.25 \%$ in NHC and $0.45 \%$ in HepG2).

\section{RNA Sequencing}

In order to investigate the transcriptional changes during the iPSC to iDC transition, we performed an unbiased, genomewide analysis of transcription using next-generation sequencing. RNA sequencing was performed on three biological replicates from each of the five phases of differentiation (iPSC, DE, HS, HP, and iDC), as well as cholangiocyte cell lines (H69 and NHC) and iHCs (isolated from human 
a

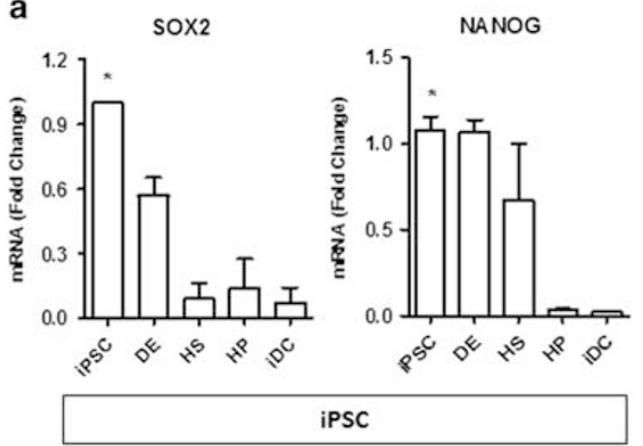

c

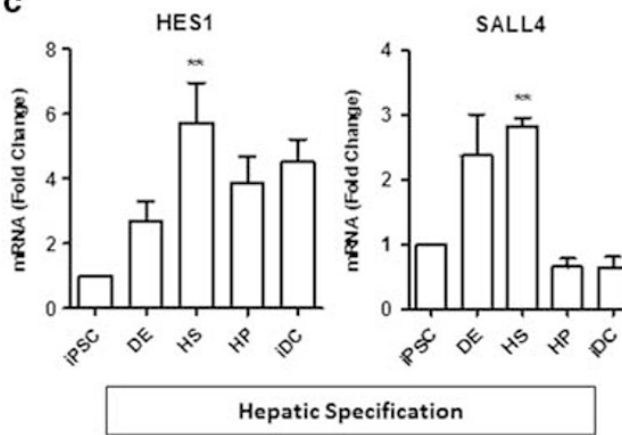

b
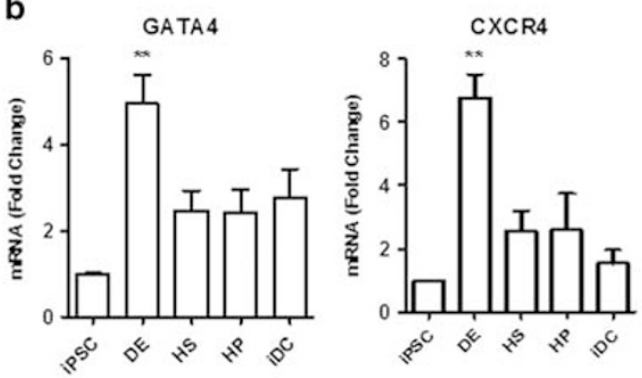

Definitive Endoderm

d

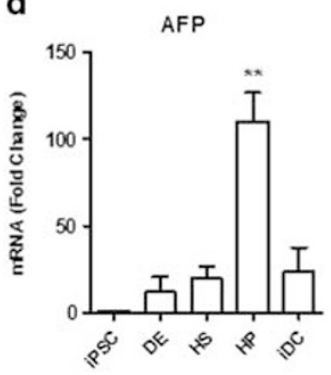

PROX1

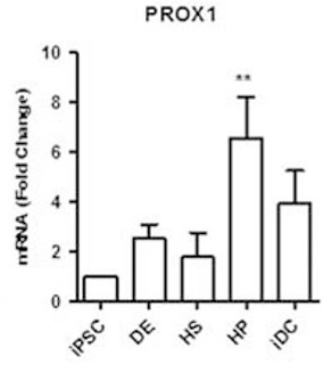

Figure 2 Molecular analysis. Real-time PCR showing expression of (a) pluripotency markers (SOX2 and NANOG) in induced pluripotent stem cells (iPSCs), followed by (b) expression of definitive endoderm markers (GATA4 and CXCR4), (c) hepatic specification markers (HES1 and SALL4), and (d) hepatic progenitor markers (AFP and PROX1). ${ }^{*} P \leq 0.05$ compared with iPSC-derived cholangiocyte (iDC) and ${ }^{*} P \leq 0.05$ compared with iPSC. All data are normalized to GAPDH and iPSC expression is set to 1.0 .

a

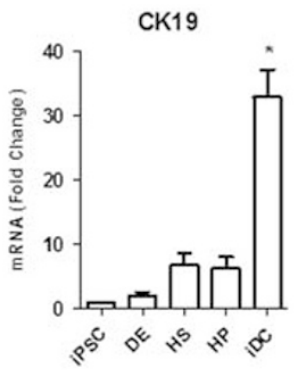

PKD2

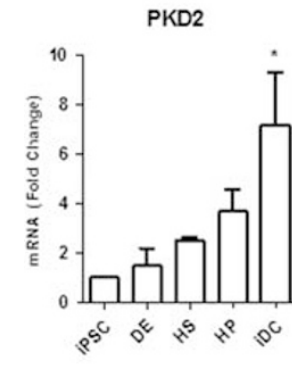

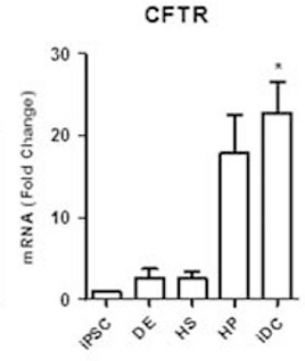

CK7

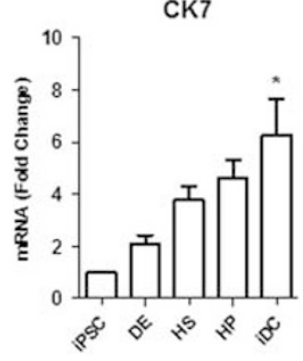

CFTR

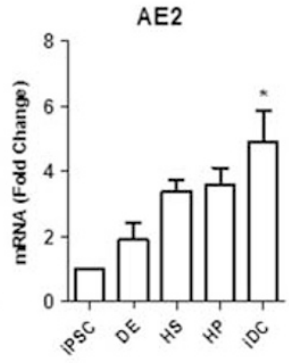

Cholangiocyte Markers

b
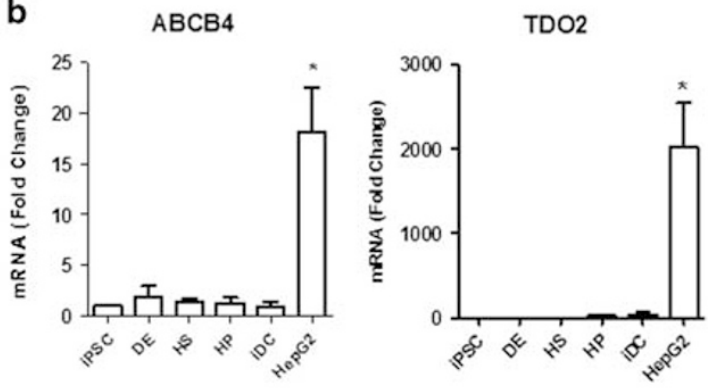

Albumin
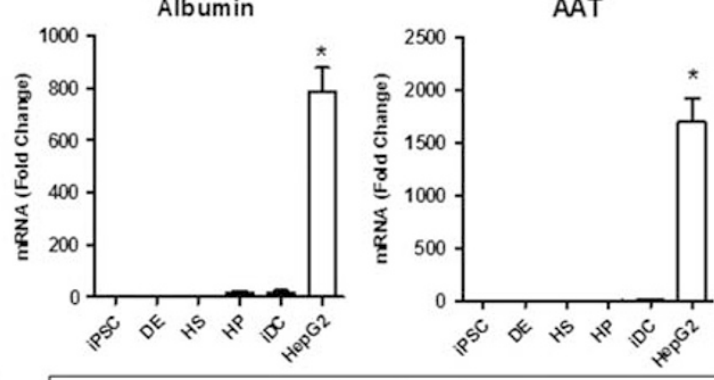

Hepatocyte Markers

Figure 3 Cholangiocyte gene expression. Real-time PCR shows that (a) induced pluripotent stem cell (iPSC)-derived cholangiocytes (iDCs) are enriched in the cholangiocyte markers cytokeratin (CK)-19, CK-7, CFTR, PKD2, and AE2. iDCs lack expression of (b) hepatocyte markers, ABCB4, albumin, $a$-1-antitrypsin (A1AT), and tryptophan 2,3-dioxygenase (TDO2), ${ }^{*} P \leq 0.05$ compared with iPSC. All data are normalized to GAPDH and iPSC expression is set to 1.0 . 
a

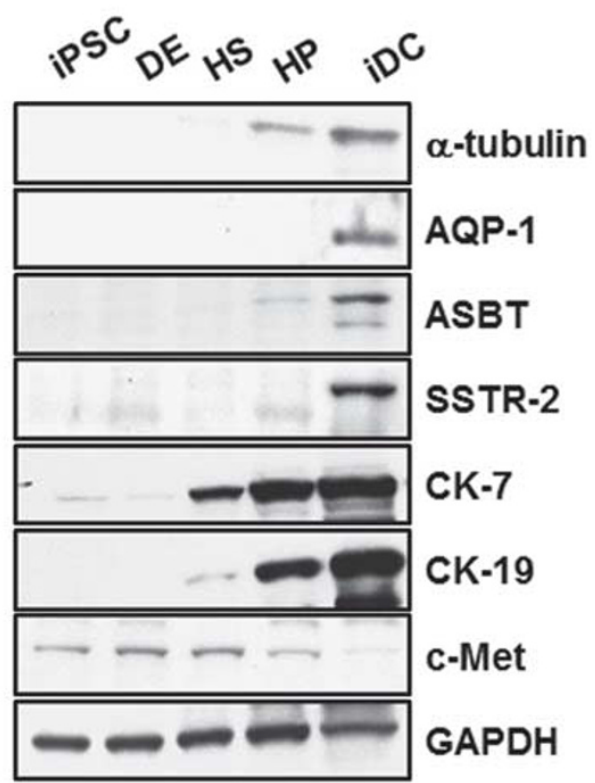

b
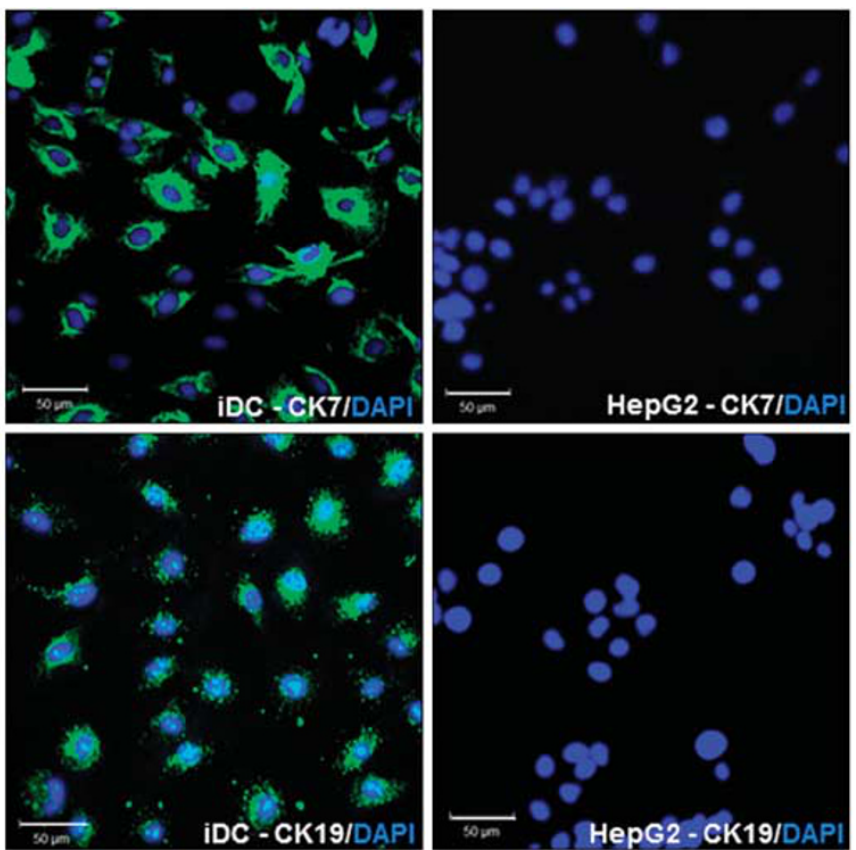
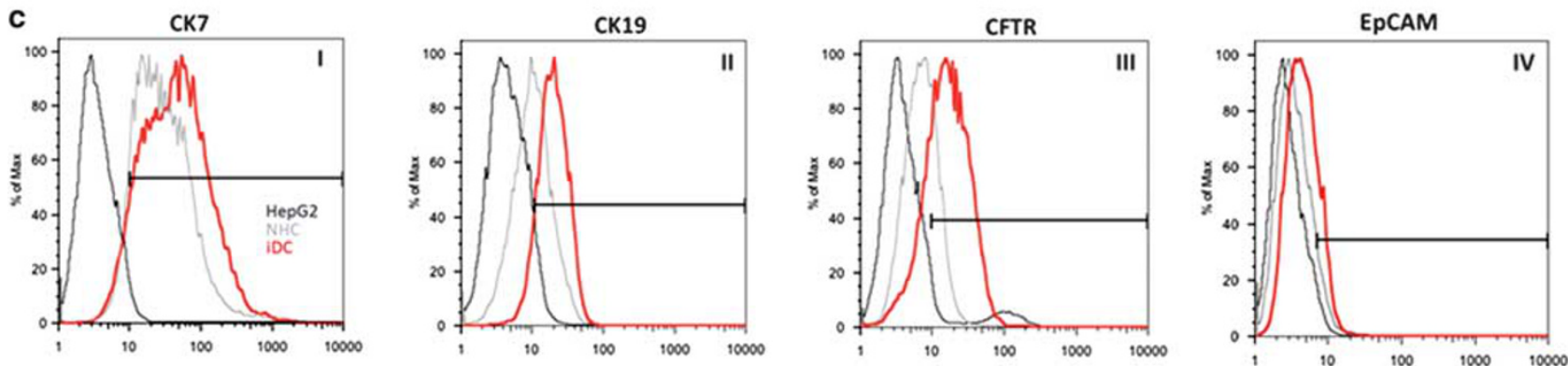

Figure 4 Expression of cholangiocyte proteins. (a) Western blotting shows enrichment of multiple, functionally relevant biliary proteins in induced pluripotent stem cell (iPSC)-derived cholangiocytes (iDCs) including acetylated $a$-tubulin, AQP-1, ASBT, SSTR-2, cytokeratin-7 (CK-7), and CK-19, while c-Met was not significantly detected. (b) iDCs were stained for CK7 and CK19 by immunofluorescence (left panels). HepG2 hepatocytes showed no staining for either marker (right panels). (c) Flow cytometry analyses of choalngiocyte markers, including CK-7, CK-19 and CFTR (panels I, II and III, respectively) and hepatic endoderm marker EpCAM (panel IV). Scale bars, $50 \mu \mathrm{m}$.

surgical resection specimens). Principal component analysis shows a smooth transition in global transcriptional changes from iPSC (red) to iDC (gold), with a trajectory toward the transcriptional profiles of the human cholangiocytes (Figure 5a). The iDC cells remain in close proximity to the HP phase on the PCA plot, as many progenitor markers are known to remain positive in cholangiocytes. However, they have also further differentiated from the progenitor phase toward cholangiocytes. Interestingly, iDCs appear to have an intermediate molecular phenotype between the freshly isolated iHCs and the cholangiocyte cell lines, which have been in long-term cell culture. Differential expression analysis confirms the similarities between iDCs and cholangiocyte cell lines. A Venn diagram (Figure 5b) showing similarly regulated genes (ie, upregulated, unchanged, or downregulated, relative to iPSC) demonstrates that among the 17725 genes analyzed, 11031 are similarly regulated compared with $\mathrm{H} 69$ cells (62.2\%) and 11506 are similarly regulated compared with NHCs (65.0\%). 9155 genes are similarly regulated relative to both groups $(21.3 \%)$. This later group of genes (i.e., the center of the Venn Diagram) may represent a cholangiocyte signature of critical gene regulation that occurs during the iPSC to cholangiocyte transition. We also observed that as cells progress through differentiation, they become progressively less similar to iPSC, as the number of differentially expressed genes, relative to iPSC, increases (DE: 1898, HS: 3017, HP: 6256, iDC: 7941), as do the magnitude of the changes seen on Volcano plots (Supplementary Figure 4). Furthermore, the number (iDC: 7941, H69: 9724, NHC: 9295) and magnitude (Supplementary Figure 4) of differentially expressed genes are ultimately similar to both cholangiocyte cell lines. A 

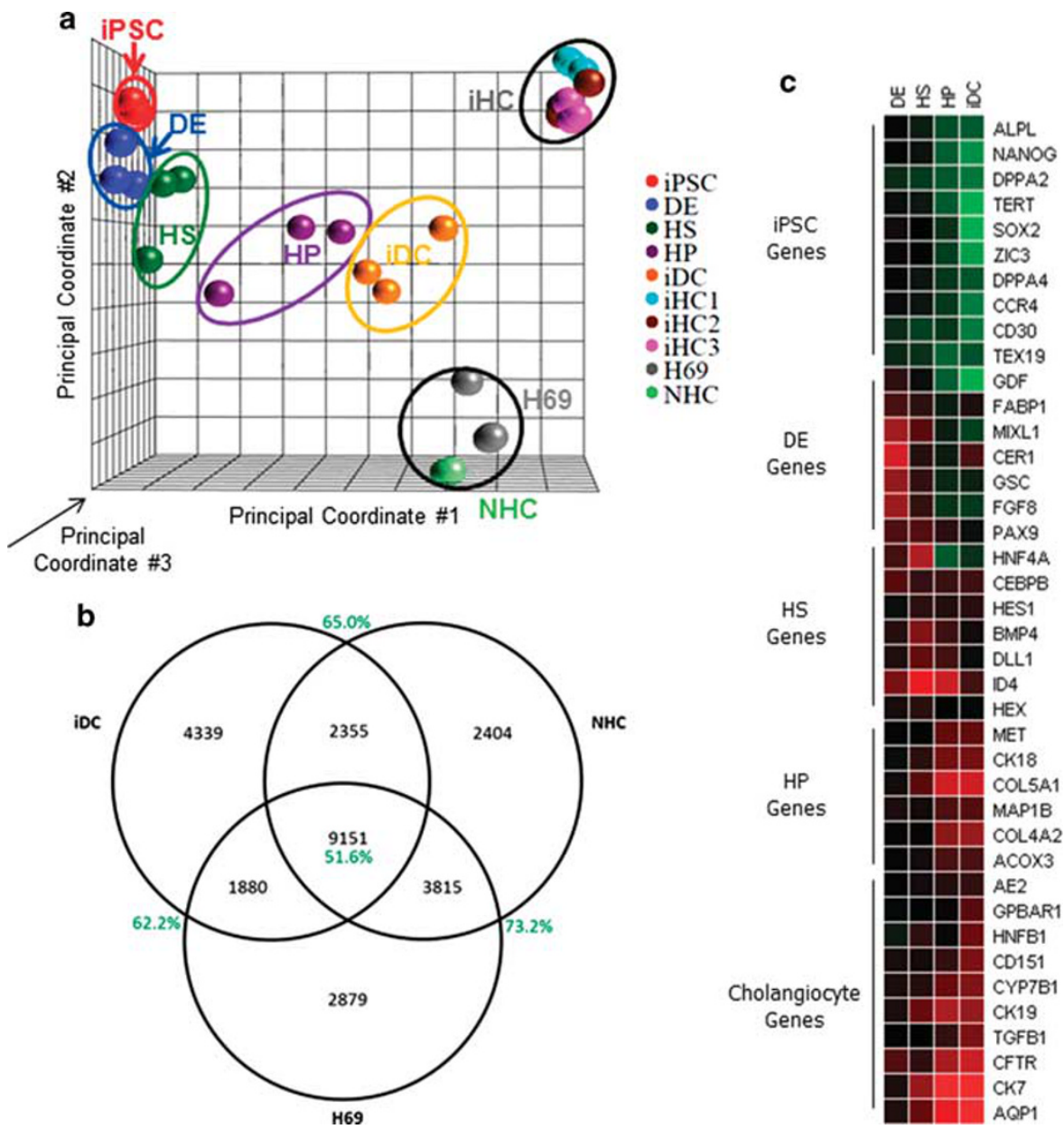

Figure 5 RNA sequencing. Sequencing of the entire transcriptome was performed at each phase of differentiation and compared with the transpcriptome of cholangiocyte cell lines and isolated human choloangiocytes. (a) Principal component analysis provided a three-dimensional graphical representation of the gene expression clustering between groups based on three mathematically defined principal components. Triplicate samples are contained within the ovals. We see a smooth transition from induced pluripotent stem cell (iPSC; red) to iDC (gold) with a trajectory toward the H69 (gray), NHC (green), and isolated human cholangiocyte (iHC; light blue, brown, and purple) cholangiocytes. (b) Differential expression analysis, performed relative to iPSC, displayed as a Venn diagram, confirms similarly regulated genes between iDCs, H69 cells, and NHCs. (c) A heatmap showing foldchange relative to iPSC demonstrates appropriate enrichment in developmental genes as differentiation progresses.

supervised interrogation of the expression profiles at each phase of differentiation reveals a heat map showing appropriate enrichment in prototypical developmental genes at each phase (Figure $5 \mathrm{c}$ ). Genes associated with pluripotency are progressively downregulated (green), while characteristic cholangiocyte genes are increasingly activated (red). Overall, these data suggest that the transcriptome of iDCs has gradually diverged from that of iPSC and has become progressively more cholangiocyte like, and that our protocol may represent a legitimate in vitro model of biliary differentiation.

\section{iDCs Form Primary Cilia and Have Intact Calcium Signaling} In order to assess the functionality of iDCs, we first assessed their ability to form primary cilia. Primary cilia are important functional organelles known to be present on the apical membrane of cholangiocytes, but not on hepatocytes. Primary cilia act as osmoreceptors and mechanoreceptors that can detect and transmit multiple signals influencing cholangiocyte function. iDC cells cultured at confluence for 7 days and serum starved for $24 \mathrm{~h}$ (conditions known to promote cilia formation in cholangiocytes) were able to form primary cilia as demonstrated by immunofluorescence for acetylated $\alpha$ tubulin (Figure 6a). At the ultrastructural level, scanning electron microscopy also revealed the presence of mature primary cilia (Figure 6b). As calcium signaling can be activated in cholangiocytes by purinergic receptors on primary cilia, ${ }^{39}$ we measured calcium signaling in response to ATP, as assessed by fluorometric calcium assays. These demonstrated rapid and robust responses to the agonist, similar to a cholangiocyte cell line (Figure 6c) and consistent with other reports of stem cell-derived cholangiocytes. ${ }^{22}$ 
a

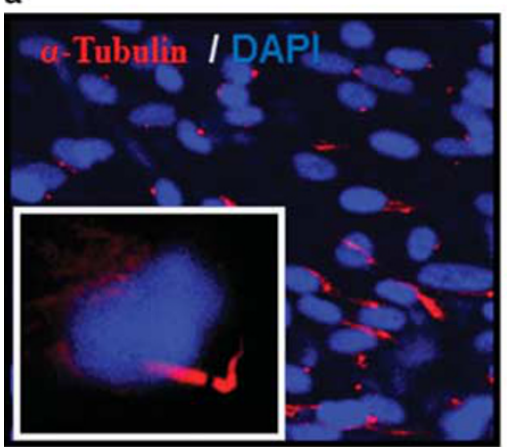

d

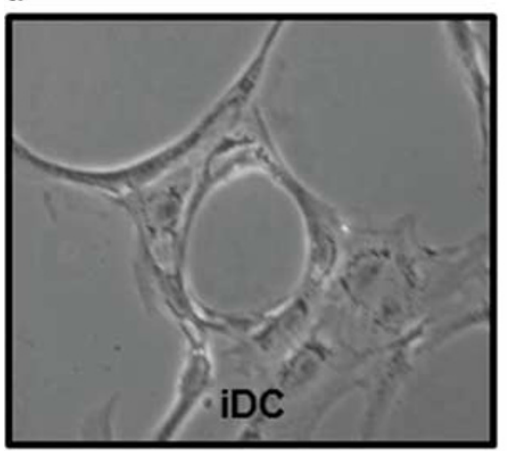

g

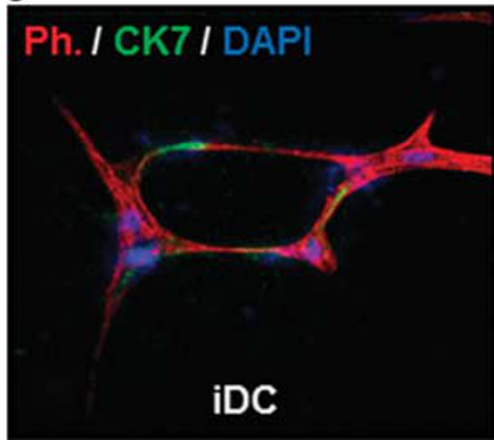

b

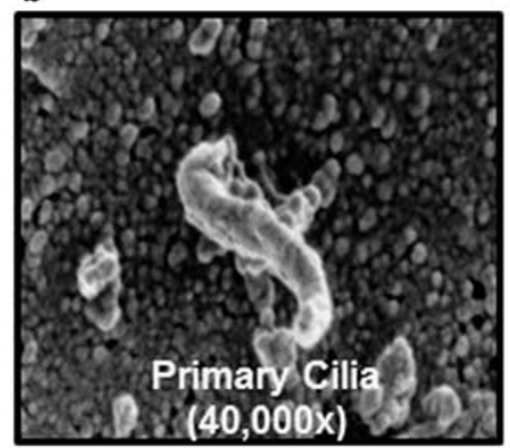

e

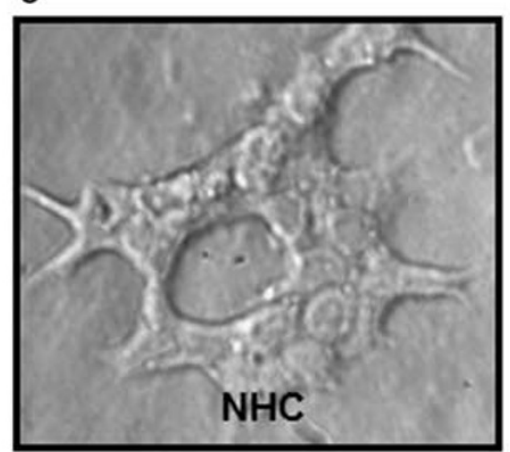

h

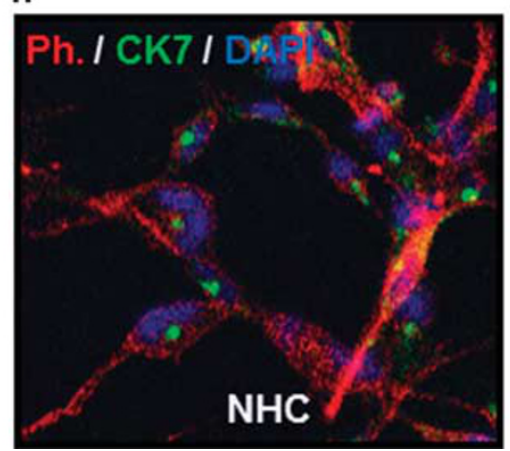

C

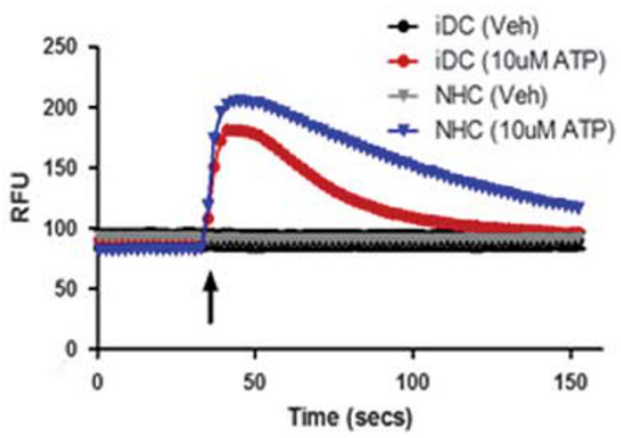

f

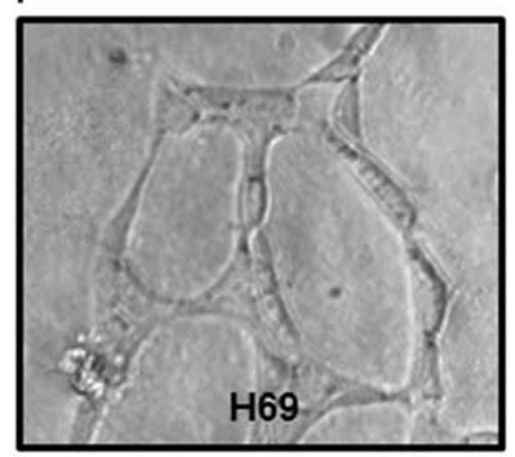

i

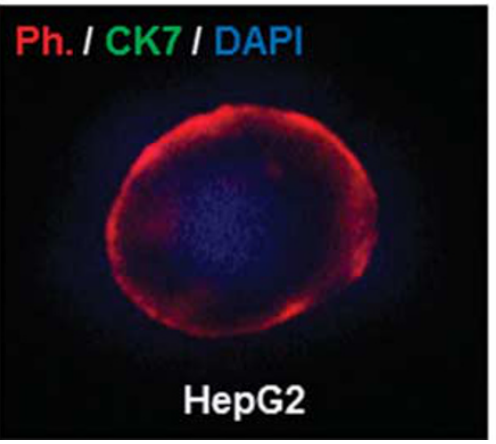

Figure 6 Functional characteristics of induced pluripotent stem cell-derived cholangiocytes (iDCs). iDCs were able to form primary cilia as shown by (a) immunofluorescence for acetylated $a$-tubulin (red) and DAPI (4'6-diamidino-2-phenylindole dihydrochloride; blue), or by (b) scanning electron microscopy. (c) iDCs activate intracellular calcium signaling in response to ATP. (d) iDCs form round duct-like structures with a luminal space when cultured for 7 days in a three-dimensional (3D) type I collagen/Matrigel culture system, similar to (e) NHC cells and (f) H69 cells. (g) Co-staining with phalloidin (red), cytokeratin (CK)-7 (green) and DAPI (blue) confirms CK-7 positivity in the duct-like structures, similar to (h) NHC cells, but different from (i) HepG2 hepatocytes.

\section{iDC Form Duct-like Structures in 3D Culture}

When iDC were cultured for 7 days in a 3D collagen/Matrigel culture system, they were able to form round duct-like structures (Figure 6d) with a branching morphology and a central cavity, features that are typical of other cholangiocyte cells in culture (Figure 6e and f). Co-staining for phalloidin (to show cellular structure) and CK7 shows that the cells retain cholangiocyte markers in this configuration (Figure 6g), similar to NHC cells (Figure 6h) but distinct from HepG2 hepatocytes (Figure 6i). Together, the above data suggest that our differentiation method can be used to generate functional adult cholangiocytes from human iPSC.

\section{iDCs Engraft Within Mouse Liver In Vivo}

We next sought to evaluate the functionality of iDC in vivo, by assessing their engraftment potential and behavior after delivery to the biliary system in a mouse model. We developed a microsurgical technique for retrograde intrabiliary infusion in the mouse as a means to deliver the cells from our last step of differentiation (Figure 7a). In this model, after clamping of the distal common bile duct, the gallbladder was dissected free from the liver and two sutures are placed around the cystic duct. Around $10^{6}$ cells (some labeled with GFP) were then administered via a small angiocatheter followed by a completion of a cholecystectomy. Three days 
a

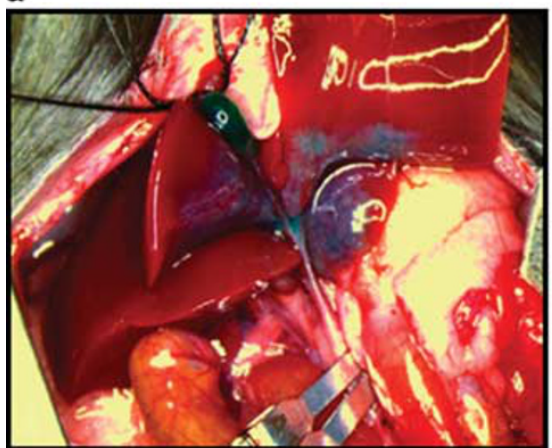

d

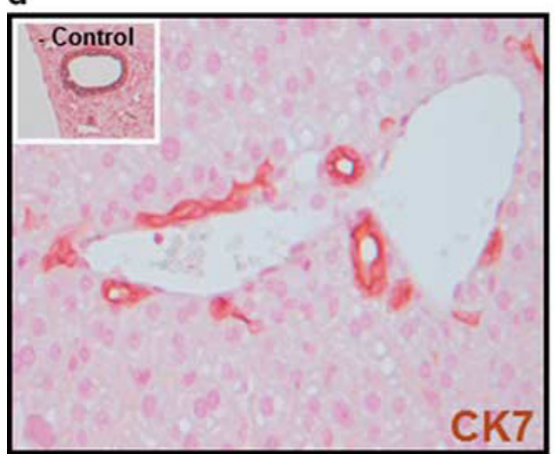

b

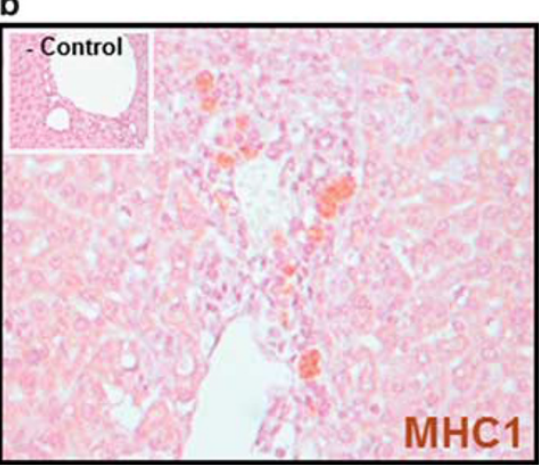

e

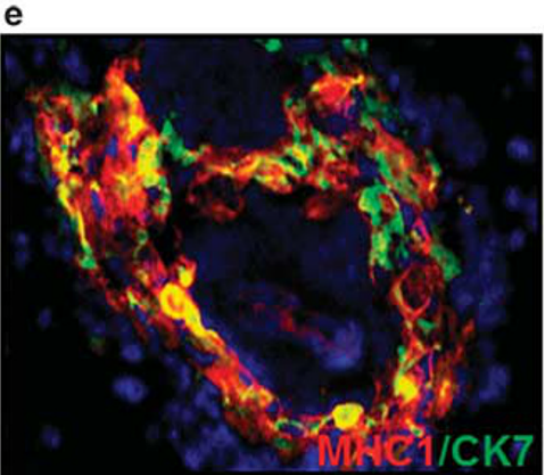

c

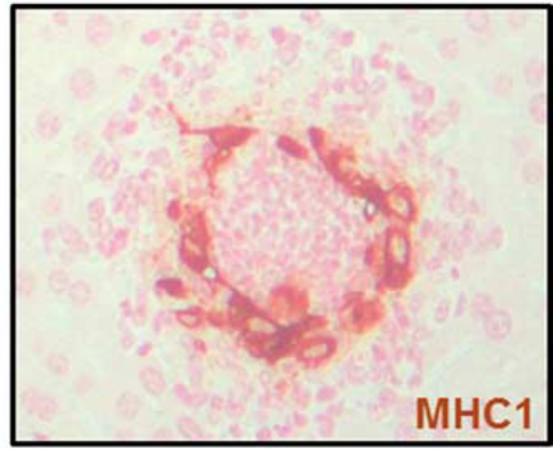

f

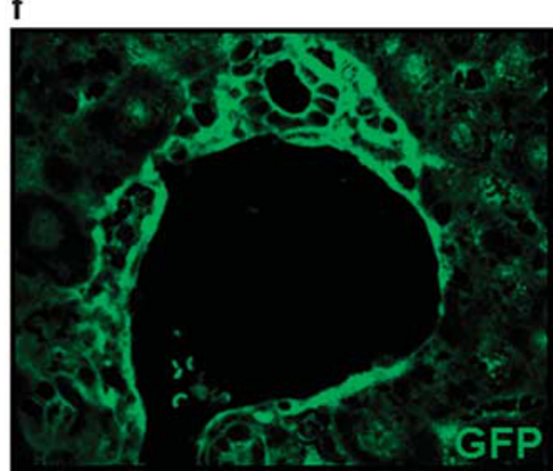

Figure 7 Retrograde intrabiliary infusion. (a) Retrograde infusion of induced pluripotent stem cell-derived cholangiocytes (iDCs) was accomplished by microsurgical cannulation of the cystic duct with temporary occlusion of the common bile duct. Engraftment was analyzed by (b, $\mathbf{c}$ ) staining for human class 1 major histocompatibility complex (MHC; inset shows a cell-free control injection) or (d) cytokeratin (CK)-7 (inset shows a cell-free control injection), by (e) dual-label immunofluorescence for MHC1 (red) and CK-7 (green), and if cells are pre-labeled with green fluorescent protein (GFP), by (f) confocal microscopy for GFP.

after cell transplantation, the liver was collected and assessed for cell engraftment and morphology by immunohistochemical staining for human MHC1 (Figure 7b and c) and CK7 (Figure 7d), by dual-label immunofluorescence microscopy for MHC1 and CK7 (Figure 7e), and by confocal microscopy for GFP expression (Figure 7f). The human iDCs engrafted within immune competent murine liver in vivo within existing portal tracts (Figure 7a) and were able to form peri-portal de novo duct-like structures (Figure 7c). Evidence of acute cellular rejection confirmed that the cells were able to engraft and provoke an immune response, as expected. The cells maintain CK7 positivity in this configuration, similar to the native mouse bile ducts (Figure 7d), but can be definitvely distinguished from the native bile ducts using dual-label immunofluorescence, which confirms co-localization of MHC1 and CK7 (Figure 7e). Developing cellular clusters without a fully mature central lumen were also observed (Supplementary Figure 5). Furthermore, GFP-labeled iDCs can be followed using confocal microscopy (Figure 7f), which also demonstrated engraftment within and among existing bile ducts.

\section{DISCUSSION}

Cholangiocyte pathophysiology has been studied in earnest now for several decades. Early basic science investments to develop unique in vitro tools ${ }^{40-42}$ as well as new animal models ${ }^{43}$ have allowed the field to realize enormous advances in our understanding of normal and pathological cholangiocyte functions and has paid dividends in terms of mechanistic insights into various cholangiopathies. Despite these remarkable advances, most of the cholangiopathies remain biologically enigmatic and clinically problematic without definitive treatment strategies other than liver transplantation when the diseases reach their end stage. In this context, the current study outlines a novel protocol for the reproducible differentiation of pluripotent stem cells to a mature and functional cholangiocyte phenotype. We have generated and fully characterized iDCs in vitro, analyzed the entire transcriptome by RNA sequencing, and for the first time we demonstrate that stem cell-derived cholangiocytes can engraft within mouse liver in vivo. The iDC protocol represents an in vitro model of biliary differentiation that appears to recapitulate several aspects of biliary development and results in a scientific platform that may be useful to mechanistically test developmental hypotheses. The system should also allow us to model biliary disease in an individualized manner, to test therapeutic compounds in vitro and to move toward cellbased therapies for the biliary system (Figure 8).

Research by several groups is now beginning to more fully elucidate many of the mechanisms that regulate and define biliary development. ${ }^{23-26}$ The foregut endoderm undergoes 


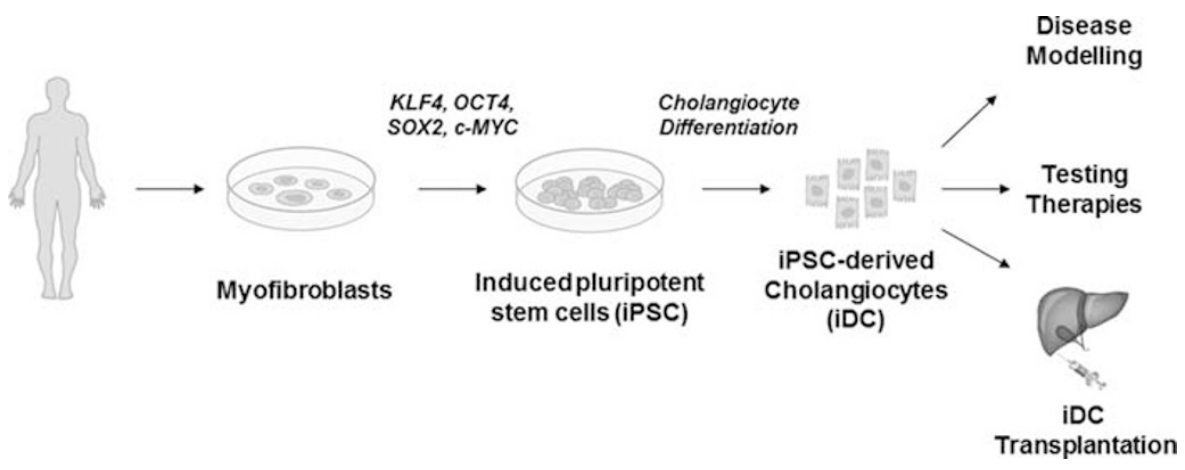

Figure 8 Development and potential uses for induced pluripotent stem cell (iPSC)-derived cholangiocyte (iDC). The flowchart outlines the development of iPSC from myofibroblasts, differentiation to iDCs, and potential downstream applications for the cells.

HS under the influence of FGF from the cardiac mesoderm and BMP from the septum transversum. Thereafter, the primitive hepatoblasts migrate into the septum transversum toward primitive portal venous structures. Hepatoblast proliferation and maturation follow, as Wnt signaling is activated via interactions with endothelial cells and stellate cells. There appears to be a gradient of Notch ligands, TGF- $\beta$, and other molecules that results in the formation of a periportal ductal plate of immature cholangiocytes. Cholangiocyte maturation, proliferation, and morphogenesis into mature tubular structures then follow in this area, while the remaining, non-peri-portal hepatoblasts move toward a hepatocyte fate instead. Accurate in-vitro platforms for modeling the developmental biology of the biliary tree have been lacking and our system was designed to recapitulate several aspects of embryologic bile duct development. As many cholangiopathies appear to have developmental defects or abnormal epigenetic patterning events that occur during embryogenesis, such a system may allow for a more detailed understanding of the pathogenesis of biliary disease as well as development of unique therapeutic targets and approaches. Whole transcriptome analysis by RNA sequencing suggests that the transcriptional profiles identified during the iPSC to iDC transition correlate with our current understanding of biliary development. As such, the system should be useful to mechanistically study how key transcriptional events may modulate hepatic lineage specification, either in the context of liver development or during progenitor cell activation in regeneration and remodeling during disease.

Rapid advances in liver regenerative medicine including differentiation of iPSC to hepatocytes has generated enthusiasm for individualized disease modeling ${ }^{44-48}$ and cell-based therapy for hepatocellular disorders. ${ }^{29,48,49}$ The development of iDCs was an obvious and important extension of this work. In that regard, our group and others ${ }^{22}$ have now begun to simultaneously develop similar, but unique, protocols to differentiate iPSC toward cholangiocytes. These disparate protocols each appear to result in cells with features of mature cholangiocytes. It is likely that each system will ultimately have strengths and limitations for various applications. Furthermore, head-to-head comparison at the transcriptomic, proteomic, and epigenetic levels should allow us to understand the subtleties that permit a multiplicity of developmental pathways to result in mature biliary cells, while differentiation of iPSC to mature and functional hepatocytes has been notoriously difficult to achieve. One interpretation of this is that biliary differentiation may represent a 'default' pathway in iPSC derivatives, while differentiation to hepatocytes requires complex in-vivo cues that are difficult to simulate in vitro. In this way, the biliary system may be an even more attractive target for regenerative medicine than parenchymal hepatocytes. There is recent evidence of biliary cells trans-differentiating to hepatocytes in the setting of massive parenchymal loss. ${ }^{50,51}$ Furthermore, it is widely thought that HP cells residing in the terminal bile ductules may contribute to liver regeneration in the setting of disease, ${ }^{52}$ although this concept has also been recently questioned. ${ }^{53}$ Although there are unresolved controversies, work by many groups is overall demonstrating remarkable plasticity and cellular trans-differentiation between hepatic epithelial cell types in various states of disease and regeneration, and our work confirms that multiple parenchymal liver cell types can also be generated from iPSC.

In this study, we have developed a unique protocol to generate cholangiocytes from human iPSC. This process represents an in vitro model of biliary development and results in mature and functional cholangiocytes that are similar to normal human cholangiocytes. This system should allow us to test genetic and epigenetic mechanisms underlying biliary development and pathophysiology, but also is a platform for individualized modeling of biliary disease and testing of pharmacotherapies. Importantly, this study is also the first to demonstrate engraftment of stem cell-derived cholangiocytes within liver in vivo, setting the stage for eventual cell-based therapeutic options for the cholangiopathies.

Supplementary Information accompanies the paper on the Laboratory Investigation website (http://www.laboratoryinvestigation.org) 


\section{ACKNOWLEDGMENTS}

We acknowledge Vijay Shah, Nicholas LaRusso, and Gregory Gores for valuable scientific discussions, Usman Yaqoob and Patrick Splinter for technical support, and Deb Hintz for secretarial support. This work was supported by grants DK100575, DK084567, and the Loan Repayment Program from the National Institutes of Health; by the Department of Oncology and

Hematology, the Second Hospital of Jilin University, China; and by the Mayo Clinic Center for Regenerative Medicine.

\section{DISCLOSURE/CONFLICT OF INTEREST}

The authors declare no conflict of interest.

1. Lazaridis KN, Strazzabosco M, Larusso NF. The cholangiopathies: disorders of biliary epithelia. Gastroenterology 2004;127:1565-1577.

2. O'Hara SP, Tabibian JH, Splinter PL, LaRusso NF. The dynamic biliary epithelia: molecules, pathways, and disease. J Hepatol 2013;58: 575-582.

3. Kochhar G, Parungao JM, Hanouneh IA, Parsi MA. Biliary complications following liver transplantation. World J Gastroenterol 2013;19: 2841-2846.

4. Mourad MM, Algarni A, Liossis C, Bramhall SR. Aetiology and risk factors of ischaemic cholangiopathy after liver transplantation. World J Gastroenterol 2014;20:6159-6169.

5. Williams MJ, Clouston AD, Forbes SJ. Links between hepatic fibrosis, ductular reaction, and progenitor cell expansion. Gastroenterology 2014;146:349-356.

6. Dianat N, Steichen C, Vallier L et al. Human pluripotent stem cells for modelling human liver diseases and cell therapy. Curr Gene Ther 2013:13:120-132.

7. Takahashi K, Yamanaka S. Induction of pluripotent stem cells from mouse embryonic and adult fibroblast cultures by defined factors. Cell 2006;126:663-676.

8. Turner R, Lozoya $\mathrm{O}$, Wang $\mathrm{Y}$ et al. Human hepatic stem cell and maturational liver lineage biology. Hepatology 2011;53:1035-1045.

9. Si-Tayeb K, Noto FK, Nagaoka M et al. Highly efficient generation of human hepatocyte-like cells from induced pluripotent stem cells. Hepatology 2010;51:297-305.

10. Sancho-Bru P, Roelandt $\mathrm{P}$, Narain $\mathrm{N}$ et al. Directed differentiation of murine-induced pluripotent stem cells to functional hepatocytelike cells. J Hepatol 2011;54:98-107.

11. Choi SM, Kim Y, Liu H et al. Liver engraftment potential of hepatic cells derived from patient-specific induced pluripotent stem cells. Cell Cycle 2011;10:2423-2427

12. Chen YF, Tseng CY, Wang HW et al. Rapid generation of mature hepatocyte-like cells from human induced pluripotent stem cells by an efficient three-step protocol. Hepatology 2012;55:1193-1203.

13. Nakamura N, Saeki K, Mitsumoto $M$ et al. Feeder-free and serum-free production of hepatocytes, cholangiocytes, and their proliferating progenitors from human pluripotent stem cells: application to liverspecific functional and cytotoxic assays. Cell Reprogram 2012;14: 171-185.

14. Yu Y, Liu H, Ikeda $\mathrm{Y}$ et al. Hepatocyte-like cells differentiated from human induced pluripotent stem cells: relevance to cellular therapies. Stem Cell Res 2012;9:196-207.

15. Aravalli RN, Cressman EN, Steer CJ. Hepatic differentiation of porcine induced pluripotent stem cells in vitro. Veterinary J 2012;194:369-374.

16. Zhu S, Rezvani M, Harbell J et al. Mouse liver repopulation with hepatocytes generated from human fibroblasts. Nature 2014;508: 93-97.

17. Szkolnicka D, Farnworth SL, Lucendo-Villarin B, Hay DC. Deriving functional hepatocytes from pluripotent stem cells. Curr Protoc Stem Cell Biol 2014;30:1G 5 1-1G 512.

18. Takayama K, Inamura M, Kawabata K et al. Generation of metabolically functioning hepatocytes from human pluripotent stem cells by FOXA2 and HNF1alpha transduction. J Hepatol 2012;57:628-636.

19. Schwartz RE, Fleming HE, Khetani SR, Bhatia SN. Pluripotent stem cellderived hepatocyte-like cells. Biotechnol Adv 2014;32:504-513.

20. Huang $\mathrm{P}, \mathrm{He} Z$, Ji S et al. Induction of functional hepatocyte-like cells from mouse fibroblasts by defined factors. Nature 2011;475:386-389.

21. Yu B, He ZY, You $P$ et al. Reprogramming fibroblasts into bipotentia hepatic stem cells by defined factors. Cell Stem Cell 2013;13:328-340.
22. Dianat $\mathrm{N}$, Dubois-Pot-Schneider $\mathrm{H}$, Steichen $\mathrm{C}$ et al. Generation of functional cholangiocyte-like cells from human pluripotent stem cells and HepaRG cells. Hepatology 2014;60:700-714.

23. Zong Y, Stanger BZ. Molecular mechanisms of bile duct development. Int J Biochem Cell Biol 2011:43:257-264.

24. Strazzabosco M, Fabris L. Development of the bile ducts: essentials for the clinical hepatologist. J Hepatol 2012;56:1159-1170.

25. Zong Y, Stanger BZ. Molecular mechanisms of liver and bile duct development. Wiley Interdisc Rev Dev Biol 2012;1:643-655.

26. Tanimizu N, Miyajima A. Molecular mechanism of liver development and regeneration. Int Rev Cytol 2007;259:1-48.

27. Greenbaum LE, Wells RG. The role of stem cells in liver repair and fibrosis. Int J Biochem Cell Biol 2011;43:222-229.

28. Machado MV, Diehl AM. Liver renewal: detecting misrepair and optimizing regeneration. Mayo Clin Proc 2014;89:120-130.

29. Huebert RC, Rakela J. Cellular therapy for liver disease. Mayo Clin Proc 2014;89:414-424.

30. Kudva YC, Ohmine S, Greder LV et al. Transgene-free disease-specific induced pluripotent stem cells from patients with type 1 and type 2 diabetes. Stem Cells Transl Med 2012;1:451-461.

31. Yang L, Kwon J, Popov $\mathrm{Y}$ et al. Vascular endothelial growth factor promotes fibrosis resolution and repair in mice. Gastroenterology 2014;146:1339-1350.

32. Kalari KR, Nair AA, Bhavsar JD et al. MAP-RSeq: Mayo Analysis Pipeline for RNA sequencing. BMC Bioinformatics 2014;15:224.

33. Trapnell C, Pachter L, Salzberg SL. TopHat: discovering splice junctions with RNA-Seq. Bioinformatics 2009:25:1105-1111.

34. Robinson MD, McCarthy DJ, Smyth GK. edgeR: a Bioconductor package for differential expression analysis of digital gene expression data. Bioinformatics 2010;26:139-140.

35. Huebert RC, Jagavelu K, Liebl AF et al. Immortalized liver endothelia cells: a cell culture model for studies of motility and angiogenesis. Lab Invest 2010;90:1770-1781.

36. Jin LF, Ji SH, Yang JF, Ji WZ. Notch signaling dependent differentiation of cholangiocyte-like cells from rhesus monkey embryonic stem cells. Dongwuxue Yanjiu 2011;32:391-395.

37. Akkari L, Haouzi D, Biname F et al. Cell shape and TGF-beta signaling define the choice of lineage during in vitro differentiation of mouse primary hepatic precursors. J Cell Physiol 2010;225:186-195.

38. Feng L, Pang L, Guo $Y$ et al. Hypoxia/reoxygenation up-regulates death receptor expression and enhances apoptosis in human biliary epithelial cells. Life Sci 2009;85:401-407.

39. $\mathrm{Yu}$ J, Sheung N, Soliman EM et al. Transcriptional regulation of IL-6 in bile duct epithelia by extracellular ATP. Am J Physiol Gastrointest Liver Physiol 2009;296:G563-G571.

40. Tietz PS, Chen XM, Gong AY et al. Experimental models to study cholangiocyte biology. World J Gastroenterol 2002;8:1-4.

41. Concepcion AR, Lopez M, Ardura-Fabregat A, Medina JF. Role of AE2 for pHi regulation in biliary epithelial cells. Front Physiol 2013;4: 413.

42. Walter TJ, Vanderpool C, Cast AE, Huppert SS. Intrahepatic bile duct regeneration in mice does not require $\mathrm{Hnf6}$ or Notch signaling through Rbpj. Am J Pathol 2014;184:1479-1488.

43. Fickert $P$, Pollheimer MJ, Beuers $U$ et al. Characterization of animal models for primary sclerosing cholangitis (PSC). J Hepatol 2014;60: 1290-1303.

44. Cayo MA, Cai J, DeLaForest A et al. JD induced pluripotent stem cellderived hepatocytes faithfully recapitulate the pathophysiology of familial hypercholesterolemia. Hepatology 2012;56:2163-2171.

45. $\mathrm{Xu} \mathrm{D,} \mathrm{Alipio} \mathrm{Z,} \mathrm{Fink} \mathrm{LM} \mathrm{et} \mathrm{al.} \mathrm{Phenotypic} \mathrm{correction} \mathrm{of} \mathrm{murine}$ hemophilia A using an iPS cell-based therapy. Proc Natl Acad Sci USA 2009;106:808-813.

46. Yusa K, Rashid ST, Strick-Marchand H et al. Targeted gene correction of alpha1-antitrypsin deficiency in induced pluripotent stem cells. Nature 2011;478:391-394.

47. Rashid ST, Corbineau S, Hannan N et al. Modeling inherited metabolic disorders of the liver using human induced pluripotent stem cells. J Clin Invest 2010;120:3127-3136.

48. $\mathrm{Yu}$ Y, Fisher JE, Lillegard JB et al. Cell therapies for liver diseases. Liver Transplant 2012;18:9-21.

49. Subba Rao M, Sasikala M, Nageshwar Reddy D. Thinking outside the liver: induced pluripotent stem cells for hepatic applications. World J Gastroenterol 2013;19:3385-3396. 
50. Choi TY, Ninov N, Stainier DY, Shin D. Extensive conversion of hepatic biliary epithelial cells to hepatocytes after near total loss of hepatocytes in zebrafish. Gastroenterology 2014;146:776-788.

51. He J, Lu H, Zou Q, Luo L. Regeneration of liver after extreme hepatocyte loss occurs mainly via biliary transdifferentiation in zebrafish. Gastroenterology 2014;146:789-800.
52. Miyajima A, Tanaka M, Itoh T. Stem/progenitor cells in liver development, homeostasis, regeneration, and reprogramming. Cell Stem Cell 2014;14:561-574.

53. Yanger $\mathrm{K}$, Knigin D, Zong $\mathrm{Y}$ et al. Adult hepatocytes are generated by self-duplication rather than stem cell differentiation. Cell Stem Cell 2014;15:340-349. 